\title{
Design of 325MHz Single and Triple Spoke Resonators at FNAL
}

\author{
G.Lanfranco, G. Apollinari, I. Gonin, T.Khabiboulline, F.McConologue, G.Romanov, R.Wagner
}

FNAL, BATAVIA, IL 60510, U.S.

\begin{abstract}
The proposed 8-GeV driver at FNAL is based on approximately 400 independently phased $\mathrm{SC}$ resonators. In this paper the design of $325 \mathrm{MHz}$ Spoke Resonators, two single spoke resonators $(\beta=0.22$ and $\beta=0.4)$ and a triple spoke resonator $(\beta=0.62)$, for the High Intensity Neutrino Source (HINS) front end is presented. We describe the optimization of the spoke resonators geometry, the goal being to minimize the $\mathbf{E}_{\text {peak }} / \mathbf{E}_{\text {acc }}$ and $B_{\text {peak }} / E_{\text {ace }}$ ratios. We report on the coupled ANSYS-MWS analysis on the resonators mechanical properties and power coupler RF design. The current status of mechanical design, slow tuning mechanism and cryostat are also presented.
\end{abstract}

\section{INTRODUCTION}

Recent discoveries in the field of neutrino physics and a renewed interest in the possibility of muon factories have motivated several proposals for high intensity (>1 MW) proton beams. At Fermilab, a $2 \mathrm{MW}, 8 \mathrm{GeV}$ proton linear accelerator based on approximately 400 independently phased superconducting and room temperature resonators has been proposed [1]. The proposed design of the LINAC is heavily based on the ILC technology and ILC-compatible operating frequencies (1300 MHz and $325 \mathrm{MHz}$ ). The LINAC layout has three major sections. The main LINAC from 1.3 to $8 \mathrm{GeV}$ uses $1300 \mathrm{MHz}$ ILC cavities, cryomodules, and klystrons. The $\beta<1$ section uses $1300 \mathrm{MHz}$ elliptical cavities that are similar to the ILC design. The Front End LINAC, operating at $325 \mathrm{MHz}$, uses a mixture of warm copper structures and superconducting spoke resonators modeled on those of the Rare Isotope Accelerator project. In the Front End, after a standard RFQ, room-temperature triple-spoke resonators are used to accelerate the beam from 2.5 to $10 \mathrm{MeV}$. Three types of superconducting spoke resonators, two single spoke resonators (SSR 1 at $\beta=0.22$ and SSR 2 at $\beta=0.4)$ and a triple spoke resonator (TSR at $\beta=0.62$ ) are used to accelerate protons from $10 \mathrm{MeV}$ to $400 \mathrm{MeV}$ [2].

Within the framework of the High Intensity Neutrino Source (HINS) program, we plan to build and operate a portion of the Front End (up to energies of $90 \mathrm{MeV}$ ) as a technical feasibility proof of the proposal. This paper focuses on the electromagnetic design and optimization of the Front End superconducting spoke resonators.

\section{RF DESIGN OF SPOKE CAVITIES}

The SSR1, SSR2 and TSR designs have been optimized using Microwave Studio (MWS) software. Fig. 1 and Fig. 2 show the main geometrical parameters of SSR1\&2 and TSR that were used for optimization.

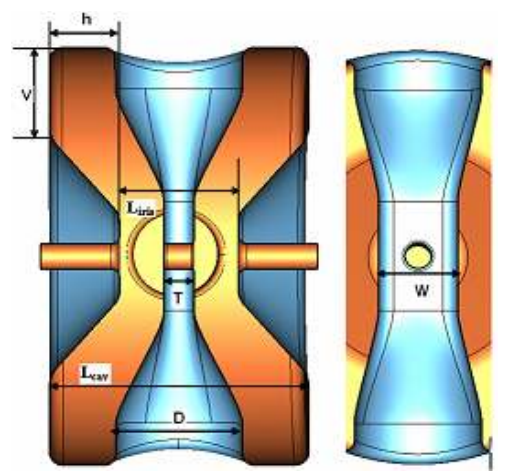

Fig. 1 - Cross section of the SSR1 and SSR2 with the main parameters used in the optimization process: Lcav - cavity length, Liris - iris to iris length, D - spoke diameter, $\mathrm{W}$ - spoke width, $\mathrm{T}$ - spoke thickness, $\mathrm{h}$ and $\mathrm{v}-$ end cup dimensions

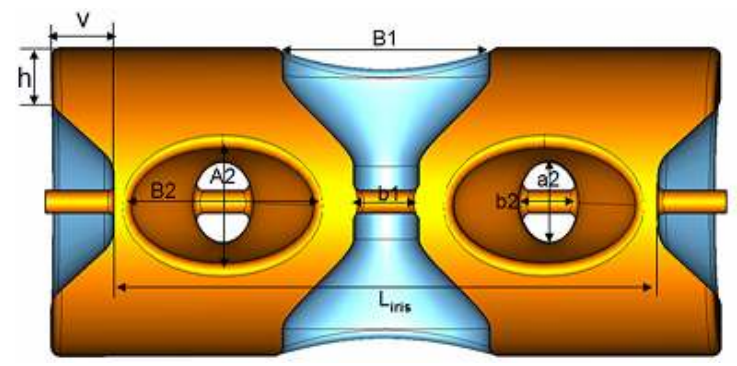

Fig. 2 - Cross section of the TSR with the main parameters used in the optimization process: Liris - iris to iris length, a1,b1,A1,B1 - ellipse half axis's of central spoke in base and axis area, a2,b2,A2,B2 - ellipse half axis's of $1 \mathrm{st}$ and $3 \mathrm{rd}$ spokes in base and axis, $\mathrm{h}$ and $\mathrm{v}-$ end cup dimensions.

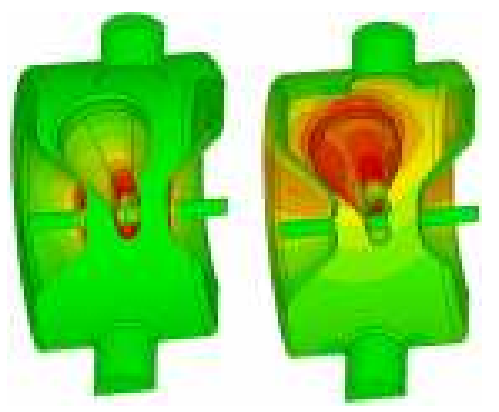

Fig. 3 - Surface electric (left) and magnetic (right) fields in SSR1. The field strength increases as the color changes from green to yellow to red.

The main criteria of the cavity RF design are to minimize the peak surface electric and magnetic field ratios $\mathrm{E}_{\mathrm{PEAK}} / \mathrm{E}_{\mathrm{ACC}}$ and $\mathrm{B}_{\mathrm{PEAK}} / \mathrm{E}_{\mathrm{ACC}}$. Surface electric and magnetic fields in optimized SSR1 and TSR cavities are shown in Fig. 3 and Fig. 4 respectively. The main RF parameters of all cavities are 
summarized in Tab. 1

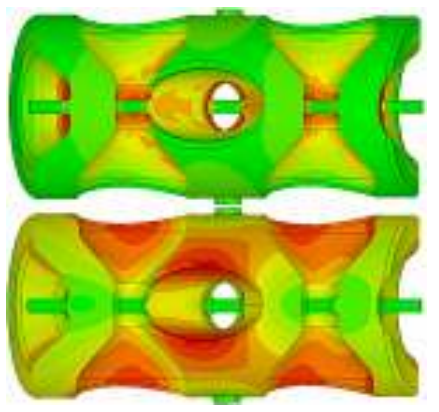

Fig. 4 - Surface electric (top) and magnetic (bottom) fields in TSR. The field strength increases as the color changes from green to yellow to red.

\begin{tabular}{|l|c|c|c|}
\hline & SSR1 & SSR2 & TSR \\
\hline $\mathrm{E}_{\text {PEAK }} / \mathrm{E}_{\mathrm{ACC}}$ & 2.55 & 2.28 & 3.22 \\
\hline $\mathrm{B}_{\mathrm{PEAK}} / \mathrm{E}_{\mathrm{ACC}}(\mathrm{mT} / \mathrm{MV} / \mathrm{m})$ & 4.87 & 4.37 & 6.85 \\
\hline $\mathrm{R} / \mathrm{Q}_{0}(\Omega)$ & 242 & 310 & 562 \\
\hline $\mathrm{G}(\Omega)$ & 78 & 82 & 97 \\
\hline \hline
\end{tabular}

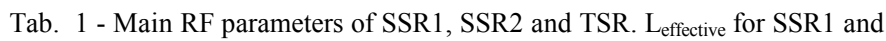
SSR2 is $2 / 3 \beta \lambda$ and for TSR $5 / 3 \beta \lambda$.

\section{Simulation Of LORENTZ DetUning}

The coupled MWS-ANSYS algorithm for static Lorentz forces detuning (LFD) factor has been developed in the following way:

1. Generation of MWS and ANSYS solid models

2. Mesh cavity volume \& structural volume (cavity \&structural facets merge at cavity wall)

3. Run MWS

4. Export pressure at merged nodes

5. Run ANSYS

6. Evaluation of frequency shift using Slater rule, see. Fig. 5.

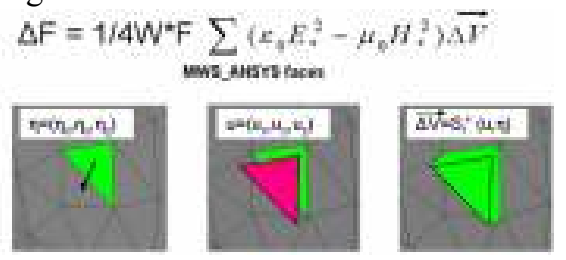

Fig. $5-\mathrm{E}^{*}$ and $\mathrm{H}^{*}$ - average amplitude of electric and magnetic fields in FE volume. $\Delta \mathrm{V}-$ the volume of prism defined by undeformed (green) and deformed (red) FE facets.

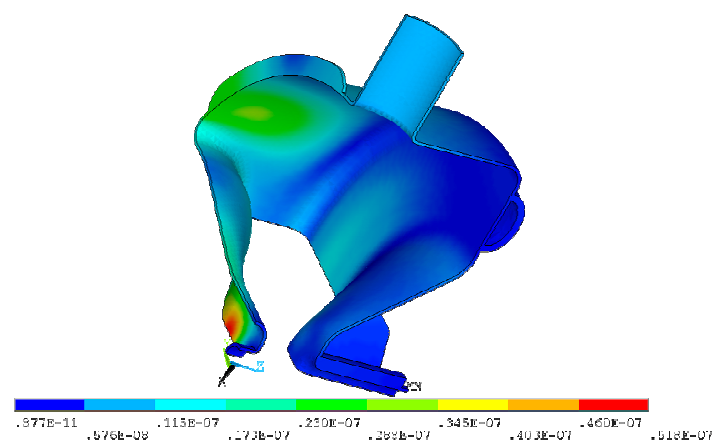

Fig. 6 - Cavity walls deformation of SSR1 due to Lorentz forces.
This algorithm has been applied to evaluation of LFD in SSR1. In process of simulations we found that adding of an additional shell ribs (see Fig. 7) reduced the detuning coefficient from $4.2 \mathrm{~Hz} /(\mathrm{MV} / \mathrm{m})^{2}$ up to $3.8 \mathrm{~Hz} /(\mathrm{MV} / \mathrm{m})^{2}$. Fig. 6 shows the deformation of SSR1 walls due to Lorentz forces. We used fix boundary conditions in LFD calculations.

\section{SSR AND SLOW Tuner Mechanical Design AND ANALYSIS}

The structural integrity of the cavity is guaranteed by a system of three types of ribs, as shown in Fig. 7 for the SSR1. Each end wall of the resonator is reinforced by a tubular rib with elliptical section and six radial daisy-like ribs. A third system of 4 ribs is present on the cylindrical shell of the cavity. A similar rib system concept is implemented for the SSR2 and TSR type cavities. Ribs are made of reactor grade niobium.

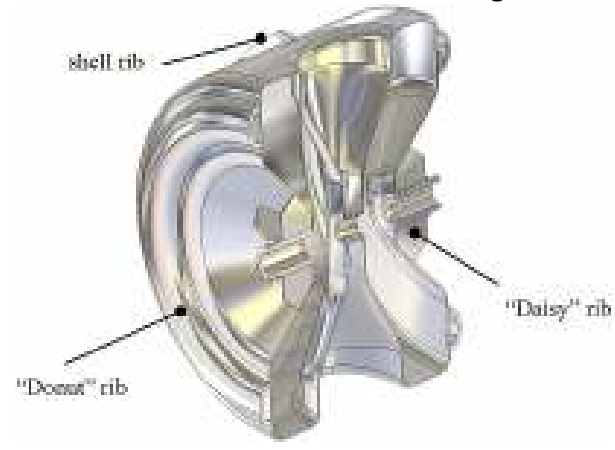

Fig. 7 - Cross section of the SSR1 structure. The stiffening ribs systems are highlighted.

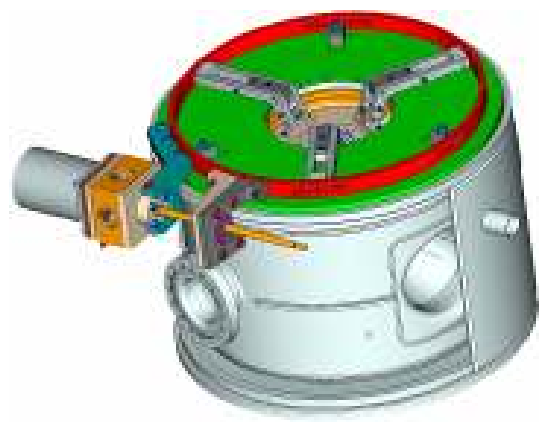

Fig. 8 - The slow tuner mechanism. One of the two He vessel shells is hidden with the purpose of showing the resonator. The slotted push/pull ring is shown is red. Each module has one slow tuner.

Such a solution allows the structure to withstand the helium pressure and limits the frequency shift caused by Lorentz force detuning while ensuring a reasonable compliance to the slow tuner action (see the FEA results of Fig. 9).

Fig. 8 gives an overview of the slow tuner mechanism, whose design is standardized for all three resonator types. The stepper motor is mounted on the outer portion of the helium vessel end wall, which has been properly modified with a bellows to allow relative motion between the inner and outer wall. The tuner, through a roller screw, puts in rotation the slotted push/pull ring allowing for the motion of three arms spaced $120^{\circ}$ apart. According to the ring rotation orientation, 
the arms will push or pull the cavity to achieve the target frequency. All contact surfaces have a tungsten disulfide (WS2) coating and the roller screw nut is lubricated with graphite. Tab. 2 summarizes some of the main slow tuner properties.

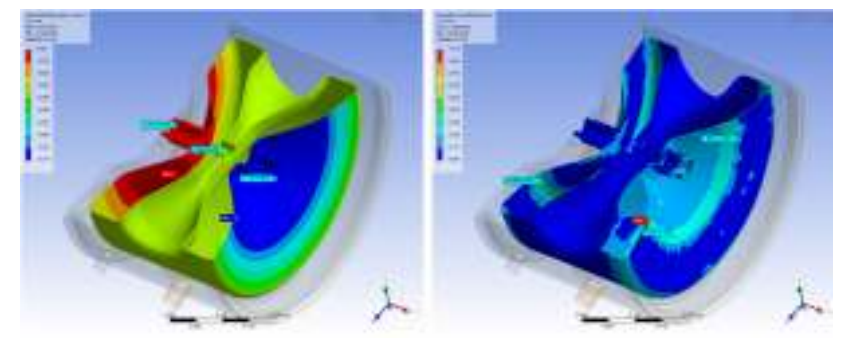

Fig. 9 - Left: longitudinal deformation $[\mathrm{mm}]$; right: von Mises stress $\left[10^{7}\right.$ Pa]. FEA boundary conditions: half structure studied (symmetry), He vessel fully constrained at fixed end wall flange (left in the pictures), $2000 \mathrm{~N}$ ( $\sim 50$ lbf) force applied at the inner end wall inside the bellows (right hand side). Max longitudinal deformation $\sim 113$ micron, von Mises stress $<12 \mathrm{MPa}$. Estimated frequency shift $\sim 60 \mathrm{KHz}$.

\begin{tabular}{||l|r||}
\hline \hline Kinematics & \\
\hline Push/pull ring rotation & $\pm 8.7^{\circ} / 37 \mathrm{~mm}$ \\
\hline$\delta_{\text {SSR1 }}$ (SSR1 longitudinal def.) & $\pm 264 \mu \mathrm{m}$ \\
\hline Frequency-deformation relation & $0.5 \mathrm{KHz} / \mu \mathrm{m}$ \\
\hline Frequency range & $\pm 131.8 \mathrm{KHz}$ \\
\hline Tuning sensitivity & $14 \mathrm{~Hz} /$ microstep \\
\hline Dynamics & $4643 \mathrm{~N}$ \\
\hline F on SSR1 to get $\delta_{\mathrm{SSR} 1}$ & $551 \mathrm{~N}$ \\
\hline F on each arm @ ring interface & $830 \mathrm{~N}$ \\
\hline F on roller screw (axial) & $63.7 \mathrm{~N} \mathrm{~cm}$ \\
\hline Torque required & \\
\hline
\end{tabular}

Tab. 2 - Slow tuning kinematics and dynamics for the SSR1. Safety factor of 10 on friction coefficients: $\mu_{\mathrm{WS} 2}=0.3, \mu_{\mathrm{screw}}=0.1$. The load due to the $600 \mu \mathrm{m}$ He pressure induced displacement has been considered as well.

\section{POWER COUPLER Design}

The universal antenna power coupler is used for all three types of $325 \mathrm{MHz}$ superconducting spoke cavities. It is installed in the mid-plane of the cavity perpendicular to spoke, where the minimum of the surface magnetic field and a strong electric field is located, as shown in Fig. 10. Fig. 11 shows the pulsed power dissipation on the coupler tip as a function of the external Q of the coupler .

As shown in Fig. 12, the design of the coupler includes two vacuum windows, as in the part of the LINAC with ILC cavities. Benefits of using two windows are:

- Double protection of the high vacuum in the cavity.

- Allows assembly of the cold part of the coupler to the cavity in a very clean environment before installation of the cavity into the cryomodule.

Two full size prototypes of the power coupler are in production. A test station is being designed that will allow the operation of two couplers at the highest power levels and processing them when necessary.

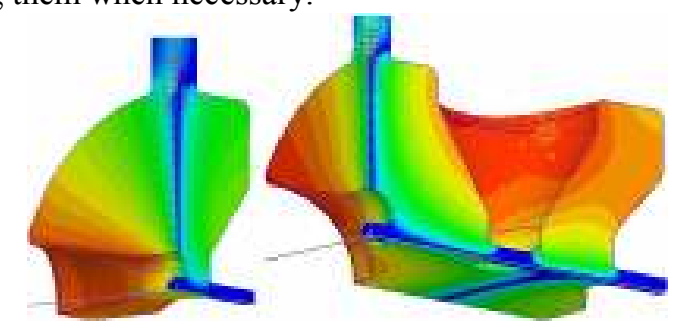

Fig. 10 - Magnetic field distribution in SSR1 and TSR with power couplers.

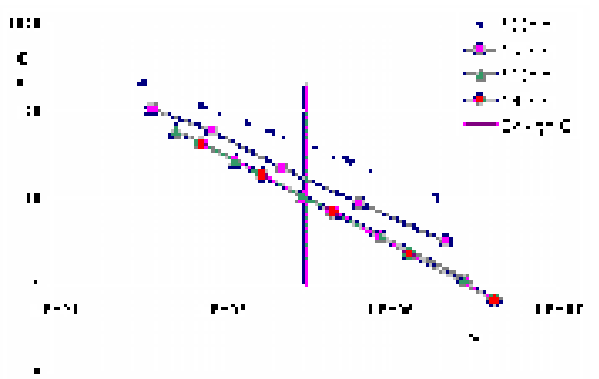

Fig. 11 - Pulsed power dissipation on the coupler tip .vs. external Q of the coupler at different positions of the coupler.

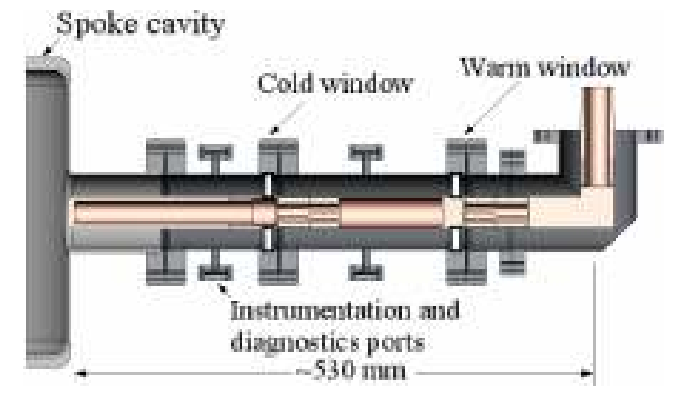

Fig. 12. Mechanical design of the double window power coupler for the single spoke cavity.

\section{CONCLUSION}

We presented the RF and mechanical designs of the superconducting spoke cavities intended for the proposed 8$\mathrm{GeV}$ proton LINAC at FNAL. The electromagnetic optimization was coupled with mechanical studies to develop designs of stiffening ribs and a slow tuner. The power coupler design is universal for all cavities. The construction of an SSR1 cavity prototype is in progress.

\section{ACKNOWLEDGEMENTS}

The authors wish to acknowledge the help received from K.Shepard, J.Fuerst and P. Ostroumov from ANL.

\section{REFERENCES}

[1] G. W. Foster and J. A. MacLachlan, Proc. of the LINAC'02, Gyeongju, Korea, August 2002, p. 826.

[2] K.W. Shepard, P.N. Ostroumov, and J.R. Delayen, Phys. Rev. ST Accel. Beams 6, 080101 (2003). 